\title{
ON A GENERALIZED MOMENT PROBLEM
}

\author{
J. S. HWANG
}

AbSTRACT. The well-known Müntz-Szász theorem asserts that the sequence of powers $x^{n} p$ is complete on $[a, b]$, where $a \geqslant 0$, if and only if

$$
\sum_{p=1}^{\infty} \frac{1}{n_{p}}=\infty \text {, where } 0<n_{1}<n_{2}<\cdots \text {. }
$$

Let $f(x)$ be absolutely continuous, $\left|f^{\prime}(x)\right| \geqslant k>0$, and $f(a) f(b) \geqslant 0$. We prove that under the assumption (1) the sequence $\left\{f(x)^{n} p\right\}$ is complete on $[a, b]$ if and only if $f(x)$ is monotone on $[a, b]$.

1. Introduction. Let $L[a, b]$ be the space of all summable functions defined on the finite interval $[a, b]$. As usual (see R. P. Boas [2, p. 234]), a sequence of functions $f_{n}(x)$ is complete on $[a, b]$ if for any $g \in L[a, b]$, the equalities

$$
\int_{a}^{b} f_{n}(x) g(x) d x=0, \quad n=1,2, \ldots,
$$

imply that $g(x)=0$, a.e. (almost everywhere) on $[a, b]$.

The Müntz-Szász theorem can be extended as follows by elementary methods.

THEOREM 1. Under the assumption (1), the sequence of polynomials $(x(1-x))^{n_{p}}$ is complete on $[0, \delta]$ if and only if $\delta \leqslant \frac{1}{2}$.

Naturally, we may ask whether the polynomial $x(1-x)$ can be replaced by a more general function. In this note, we settle such an extension of the Müntz-Szász theorem as well as a related theorem of Picone; see Boas [1].

2. Müntz-Szász theorem. To formulate the desired theorem, we first observe that the derivative of the function $f(x)=x(1-x)$ satisfies $f^{\prime}(x)=1-2 x \geqslant 1-2 \delta>0$ for every $x \in[0, \delta]$, where $\delta<\frac{1}{2}$. This gives the motivation for the following extension of the Müntz-Szász theorem.

THEOREM 2. Let $f(x)$ be a function absolutely continuous on a finite interval $[a, b]$, $f(a) f(b) \geqslant 0$, and $\left|f^{\prime}(x)\right| \geqslant k>0$, a.e. on $[a, b]$; then under the assumption (1) the sequence $\left\{f(x)^{n_{p}}\right\}$ is complete on $[a, b]$ if and only if $f(x)$ is monotone on $[a, b]$.

Proof. Sufficiency. We may, without loss of generality, assume that the function $y=f(x)$ is montonically increasing on $[a, b]$. Let $x=f^{-1}(y)$ be the inverse function

Received by the editors December 2, 1980 and, in revised form, March 31, 1981

1980 Mathematics Subject Classification. Primary 30B60, Secondary 26A48.

Kev words and phrases. Completeness, moment problem, absolutely continuous and monotone function

'The author acknowledges support from H. L. Jackson's NSERC Grant (A7322) when the author visited McMaster University. 
of $f(x)$; this function is montonically increasing on $[f(a), f(b)]$. Then by the identity $y=f\left(f^{-1}(y)\right)$ and the hypothesis $\left|f^{\prime}(x)\right| \geqslant k>0$, a.e. on $[a, b]$, we obtain

$$
\left|f^{-1}(y)^{\prime}\right|=1 /\left|f^{\prime}(x)\right| \leqslant 1 / k, \text { a.e. on }[f(a), f(b)] \text {. }
$$

Moreover, $f(x)$ is absolutely continuous so that its derivative $f^{\prime}(x)$ is summable on $[a, b]$. It follows from the left-hand side of (2) that

$$
f^{-1}(y)^{\prime} \neq 0 \text {, a.e. on }[f(a), f(b)] \text {. }
$$

We now consider an arbitrary function $g \in L[a, b]$ for which

$$
\int_{a}^{b} f(x)^{n} g(x) d x=0, \quad p=1,2, \ldots
$$

By substituting $y=f(x)$ into (4), we find that

$$
\int_{f(a)}^{f(b)} y^{n_{p}} g\left(f^{-1}(y)\right)\left(f^{-1}(y)\right)^{\prime} d y=0, \quad p=1,2, \ldots
$$

In view of (2), we can see that the function $g\left(f^{-1}(y)\right)\left(f^{-1}(y)\right)^{\prime}$ is summable on $[f(a), f(b)]$. It follows from the Müntz-Szász theorem that this function

$$
g\left(f^{-1}(y)\right)\left(f^{-1}(y)\right)^{\prime}=0, \quad \text { a.e. on }[f(a), f(b)] \text {. }
$$

Owing to (3), we conclude that $g(x)=0$, a.e. on $[a, b]$. This proves the completeness of the sequence $\left\{f(x)^{n_{p}}\right\}$ on $[a, b]$.

Necessity. If the sequence $\left\{f(x)^{n_{p}}\right\}$ is complete on $[a, b]$ we shall prove that $f(x)$ is monotone on $[a, b]$. Suppose not; then clearly there exist points $q<r$ in $[a, b]$ such that

$$
f(q)=f(r)
$$

We then define the function

$$
\left\{\begin{aligned}
g(x) & =f^{\prime}(x), & & \text { for } q<x<r \text { and } f^{\prime}(x) \text { exists, } \\
& =0, & & \text { elsewhere. }
\end{aligned}\right.
$$

By (5) and (6) we obtain for $p=1,2, \ldots$ (integration by parts)

$$
\int_{a}^{b} f(x)^{n_{p}} g(x) d x=\int_{q}^{r} f(x)^{n_{p}} f^{\prime}(x) d x=-\int_{q}^{r} n_{p} f(x)^{n_{p}} f^{\prime}(x) d x,
$$

or

$$
\left(1+n_{p}\right) \int_{a}^{b} f(x)^{n_{p}} g(x) d x=0 .
$$

Since $1+n_{p} \neq 0$, it follows from (6) that the sequence $\left\{f(x)^{n_{p}}\right\}$ is not complete on $[a, b]$, a contradiction. This completes the proof.

We finally remark that the uniqueness theorem of Boas [1] and Mikusinski [3] can also be extended as in Theorem 2 .

\section{REFERENCES}

1. R. P. Boas, Jr., Remarks on a moment problem, Studia Math. 13 (1953), 59-61.

2. Entire functions, Academic Press, New York, 1954.

3. J. G. Mikusinski, Remarks on the moment problem and a theorem of Picone, Colloq. Math. 2 (1951), $138-141$.

Institute of Mathematics, ACademia Sinica, Taipei, Taiwan

Current address: Department of Mathematics, Michigan State University, East Lansing Michigan 48824 